Session: 1449

\title{
Teaching Local Area Networking Using Samba Instead of Windows
}

\author{
Gary D. Steffen \\ Electrical and Computer Engineering Technology \\ Indiana University - Purdue University Fort Wayne
}

\begin{abstract}
The increasing cost of computer server software, such as Windows NT/2000, can prohibit its use in laboratory settings. Furthermore, the system hardware requirements for these software packages can far exceed current institutional laboratory computer hardware. The same principal techniques for local area networking can be taught using Linux with Samba at a fraction of the cost. A look will be taken on how existing Windows NT/2000 server experiments can be replaced using Linux with Samba.
\end{abstract}

The initial presentation discusses the significance of Samba in the local area networking environment. Included is a description of history, availability, and cost of maintaining a Samba based network. Consideration will then be given on how existing Windows Server experiments can be modified to use Samba in its place. Important experimental issues such as domain controllers, shares, printing, authentication and security will be addressed. Included is local area networking experiments based solely on Samba that provide comparable educational benefits to Windows experiments.

Introduction

Local area networks (LAN) technology once thought of only for large companies is appearing in all realms of everyday life. Small computer networks pop up all over the place from large international corporate entities to private home networks used for Internet access. This broad range of applications has brought the instruction of LANs to the forefront of computer engineering technology.

Indiana University - Purdue University Fort Wayne (IPFW) has been offering a Local Area Networking course for several years. A leading problem with the instruction of such a course has been the constant update and maintenance of the Network Operating System (NOS) software. Like many universities, it's been a struggle to properly fund needed licensing and hardware requirements of these products.

Microsoft, a leader in NOS products, has always been our main tool in the education of Local Area Networks. These include the Windows based server products of NT, 2000 and XP.

"Proceedings of the 2003 American Society for Engineering Education Annual Conference \& Exposition Copyright (c) 2003, American Society for Engineering Education" 
Hardware requirements and continuing software version changes by Microsoft make the use of new technology a difficult proposition. Continually, we seem to teach one revision behind.

IPFW implemented the use of Linux with Samba in our LAN courses to help defray cost. The same principal techniques for local area networking can be taught using Linux with Samba as Microsoft at a fraction of the cost.

Samba

Samba is an Open Source Software (OSS) project first developed by Andrew Tridgell in 1991 at the Australian National University in Canberra, Australia. It is designed as a suite of programs which work together to allow workstations to access a server's filespace and printers via the SMB (Server Message Block) protocol. Initially written for UNIX, Samba runs on a variety of other network operating systems (NOS).

Samba, which comes packaged with most brands of the Linux Operating System (OS), is open source code just like Linux. The source code for both, written in $\mathrm{C}$, is always available to download, test and change. There is no cost for these products. (http://www.samba.org) Samba can redirect disks and printers to Linux disks and printers from Microsoft product workstations $(3.11,98,2000$ and XP) as well as Linux clients. There is also a generic Linux client program supplied as part of the suite which allows Linux users to use an ftp-like interface to access filespace and printers on any other SMB servers. This gives the capability for the operating system to behave much like a Windows NT/2000 server machine, only with added functionality and flexibility designed to make life easier for administration.

What can Samba do?

Price alone can't justify changing existing laboratory experiments to Samba, unless it meets or exceeds what a Microsoft NOS can do. Below, is a list of Samba functions that are comparable to those used when teaching Local Area Networking with Windows.

- Acts as a Primary Domain Controller (PDC) for the Windows client. This is the server that authenticates domain logons and maintains the security policy. A PDC manages user's accesses to a network, which includes logging on, authentication and access to the directory and shared recourses.

- Acts as a Backup Domain Controller (BDC) for the Windows client.

- Acts as a Netbios name server.

- Fully participates in NETBIOS browsing and browse master elections, including being the actual browse master.

- Contains SMB clients that allow Linux machines to access shares or printers on other SMB servers, windows machines or other samba servers.

- Contains extensions that allow Linux machines to back up files on remote SMB shares, such as Windows file servers.

"Proceedings of the 2003 American Society for Engineering Education Annual Conference \& Exposition Copyright (c) 2003, American Society for Engineering Education" 
- Provides a command line utility for limited remote administrative functionality of Windows and Samba servers.

- Provides a way to synchronize Linux and Windows passwords.

- Can have "home directories" so both Windows and Linux directories can coexist on the same server.

- Act as a print server between Windows and Linux networked printers.

- Allows Linux access to Windows files.

- Acts as a WINS server.

Linux extends the further usefulness of Samba by providing additional services such as DNS, WINS, TELNET, FTP, DHCP and APACHE.

Samba as a Primary Domain Controller

The key to configuring Samba to be a PDC is its lone configuration file: smb.conf. Unlike the Microsoft NOS with its multiple GUI interface, Samba has one text file containing all its matched pair settings. Even though Linux X-Windows has a GUI interface to SAMBA, known as SWAT, most people prefer editing the single text file.

Alteration to the smb.conf file can be done on the fly. There is no need to reboot the computer. The changes to Samba take effect when the SMB daemon refreshes itself or restarts. The file itself contains hundreds of settings most are not needed for simple implementations. The well documented file serves as a teaching tool in itself.

Adding the following section, see figure 1, to the area marked "[global]" of your smb.conf file will enable the Samba server as a PDC. Any line preceded by the "\#” is for comments only.

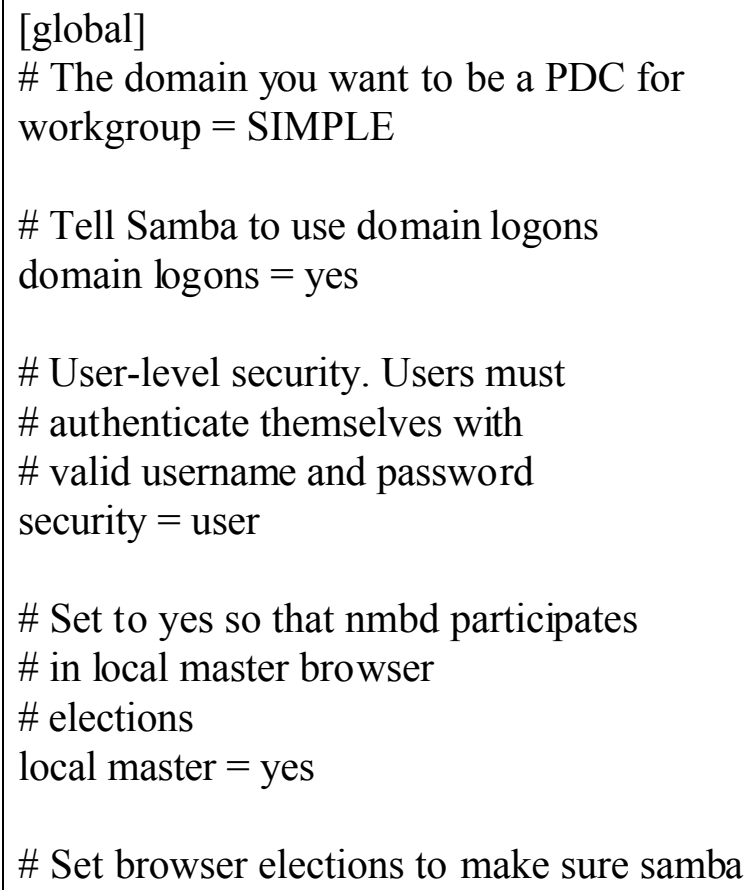


\# wins local browse master
\# elections.
os level $=65$
\# Give SAMBA an advantage in local
\# master browser elections
preferred master $=$ yes
\# Set so that SAMBA claims a unique
\# NetBIOS name identifying it as
\# a domain master
domain master $=$ yes
\# Path to Samba password file
\# Make sure only root has read/write access
smb passwd file =/usr/local/samba/smbpasswd
\# The following share is required to support
\# domain logons. The directory may be
\# created anywhere on your system. Make
\# sure the share is non-writeable and also
\# not a public share.
[netlogon]
comment = The domain logon service
path $=$ /usr/local/samba/netlogon
public = no
writeable = no

Figure 1. smb.config file settings for a Primary Domain Controller.

With samba, an account on the Linux server has to be created. This includes computer accounts for Windows NT, 2000, and XP. A token based authentication is used so the computer actually logs into the domain, not the user. The user is allowed to login as a trust. Creating the account can be done with "adduser [username]" or any other Linux method.

Once the account is added to the Linux server it now has to be added to the smbpasswd file. To create the account you can use "smbpasswd -a [username] [password]" or any other Linux method.

\section{Samba Experiments}

The Samba laboratory work covers about a six week period. Topics are introduced weekly in preparation for the laboratory setting. Each student group is equipped with their own client computer, server and communication equipment. This allows each group to manage their own independent Local Area Network.

The Samba server topics include:

- Laboratory 1 - Installing Linux and Samba

"Proceedings of the 2003 American Society for Engineering Education Annual Conference \& Exposition Copyright (C) 2003, American Society for Engineering Education" 
- Laboratory 2 - Creating and Accessing Shares

- Laboratory 3 - Samba Browsing and Disk Shares

- Laboratory 4 - Setting up a Primary Domain Controller

- Laboratory 5 - Samba Security

- Laboratory 6 - Samba Printing and Name Resolution

Each laboratory experiment is stand alone, but each is designed to build upon the previous. The students maintain the same SAMBA server throughout all the experiments. Once all laboratories are complete, the students possess a full functioning Samba server that behaves as a Primary Domain Controller.

\section{Samba Added Benefits}

From the education stand point, it's important to allow the student's access to the most important tools in Local Area Networking whenever possible. Currently students of IPFW experiment with a combination of Windows and Linux products due to corporate popularity. The Windows product used is typically always a generation old because of our limitations in computer hardware.

Windows is introduced first since most students are already experienced with the GUI interface. When first discussing Linux with Samba, most students seem intimidated since pervious Linux editions carry a reputation of being difficult to install. Most students, once over this fear, find the management aspect of Samba superior to that of Windows. They find editing the single smb.config file much easier then memorizing GUI menus under Windows. Students typically perform the Samba based experiments twice as fast as the corresponding Windows experiments.

Additional benefits of Samba are demonstrated by asking the student to do a price comparison of Linux and Windows for a small fictitious company. The study is to include software purchase price, hardware purchase price, software maintenance price and administrative cost. The student finds that the cost of maintaining Linux is far cheaper than Windows. The greatest difference is in the software purchase price and maintenance prices. A single copy of Red Hat Linux to cover all servers is \$200.00 (unlimited clients) with no recurring maintenance fee while Windows costs $\$ 1119.00$ per server (only 10 clients) with an approximate recurring cost of $25 \%$ of the original cost per year.

\section{Conclusion}

In the market of Local Area Networking, Microsoft NOS software must not be denied or overlooked. However, alternate methods of instruction based on Samba can provide comparable educational benefits to those found using Microsoft.

Students have established a long history with the Window environment. Most students introduced to Linux and Samba thrive at the opportunity to try a new technique. Even though Linux with Samba itself poses more difficulty during installation, the overall system management seems easier for students when using Samba. The lower cost of hardware and software overhead makes this a prime choice for students on their home computer network furthering the educational experience. 


\section{Bibliography}

1. Robert Eckstein, David Coillier-Brown and Peter Kelly, "Using Samba", O’Reilly \& Associates, Inc., 2000

2. Christopher R. Hertel, "Understanding the Network Neighborhood, How Linux works with Microsoft Networking Protocols", Linux Magazine, May 2001

3. Gerald Carter and Richard Sharpe, "Samba in 24 Hours", SAMS, 2001

4. http://www.samba.org

\section{GARY D. STEFFEN}

Gary currently services as an Assistant Professor in the Electrical and Computer Engineering Technology Department (CPET) at Indiana-Purdue University Fort Wayne (IPFW). Previously, he served 10 years as the Manager of Electronic and Computer Support for IPFW specializing in computer networking. He has been employed by Purdue University since 1989. Gary received his bachelors' degree in 1989 and his Maters degree in Computer Science from Ball State University in 2000. His current areas of interest include local area networking, network security and wireless networking. 